\title{
Avaliação dos Ambientes Virtuais de Aprendizagem Moodle, TelEduc e Tidia - Ae: um estudo comparativo
}

Fábio Teixeira Franciscato - PPGI/UFSM - fabiofranciscato@ yahoo.com.br

Patric da Silva Ribeiro - PPGI/UFSM - patricribeiro@uabrestingaseca.com.br

Patricia Mariotto Mozzaquatro- PPGI/UFSM - patriciamozzaquatro@gmail.com

Roseclea Duarte Medina - PPGI/UFSM - roseclea.medina@gmail.com

\section{Resumo}

Esse artigo apresenta, os resultados decorrentes da elaboração de um instrumento de avaliação e a sua aplicação nos ambientes Moodle, TelEduc e Tidia - Ae. Os questionários e as análises foram realizados por alunos de Pós - Graduação da Universidade Federal de Santa Maria, com o objetivo de proporcionar a utilização de três ambientes distintos para ampliar as experiências com ambientes virtuais de aprendizagem (AVAs) e desenvolver condições para realizar avaliações críticas. Os resultados também foram submetidos aos gestores de EAD e a equipe de TI para tomarem conhecimento das sugestões de melhorias nos AVAs utilizados na Institição (Moodle na EAD e TelEduc no ensino presencial).

Palavras-chave: educação a distância, ambientes virtuais de aprendizagem, avaliação.

\section{Evaluation of Virtual Learning Environments Moodle, TelEduc and Tidia - Ae: a comparative study}

\begin{abstract}
This article presents the results from elaboration of an evaluation instrument and its application in the Learning Environments Moodle, TelEduc and Tidia-Ae. The questionnaires and the analyses were conducted to pos graduation students from Universidade Federal de Santa Maria, with the aim of providing the use of three different Learning Environments to expand the experience with them, and develop conditions to carry out critical evaluations. The results were also submitted to the manager of distance learning and to the TI team for to know the suggestions for improvement in the Learning Environments Moodle, Teleduc, used in the university.
\end{abstract}

Keywords: distance learning, learning environments, evaluation

\section{Introdução}

Nas últimas décadas, a educação a distância (EAD) tomou um novo impulso que favoreceu a disseminação do acesso à educação em diferentes níveis e formas de interação e aprendizagens. Os processos estão, cada vez mais, se articulando através dos ambientes virtuais de aprendizagem (AVAs). No contexto acadêmico, esta realidade cria novas oportunidades para os educadores compartilharem com os alunos o acesso às informações e trabalharem de forma cooperativa. Nesse sentido, o advento das tecnologias de informação e comunicação (TICs) trouxe novas perspectivas para a EAD, levando as Instituições de Ensino, empresariais e os profissionais de instruction design a se dedicarem ao desenvolvimento de cursos a distância e AVAs. Conforme Primo (2008),

Apesar das novas tecnologias oferecerem cada vez mais recursos para a aprendizagem, a desorganização no planejamento na apresentação de um material multimídia, e a utilização inadequada de ferramentas disponíveis num AVA, podem causar desorientação do usuário que poderá até mesmo desmotivá-lo a se engajar na atividade proposta. 
Quando se decide pela utilização de um AVA é preciso estabelecer critérios coerentes que sejam mais adequados ao processo educacional. Como analisar aspectos positivos e negativos? O que deve ser relevante? Que parâmetros devem nortear esta escolha?

É nesse contexto de variáveis que se procura melhor avaliar um ambiente, podendo assim, possibilitar e promover situações de aprendizagem que mobilizem os estudantes a gerar significados e, por conseguinte, melhorar a construção de conhecimentos de forma autônoma.

A necessidade de aprofundamento no tema avaliação de AVAs, definiu os objetivos deste trabalho, que foi avaliar e comparar, a partir da análise de questionários e da observação do comportamento dos alunos com relação a utilização dos ambientes, aspectos como funcionalidades, ergonomia, usabilidade e ferramentas integrantes dos AVAs: Moodle, TelEduc e Tidia-Ae, favorecendo com isso, melhorias consideráveis nas habilidades e competências necessárias para a realização de avaliações críticas/técnicas de AVAs .

O presente artigo constitui-se de sete seções. A seção dois apresenta Ambientes Virtuais de Aprendizagem. A seção três é dedicada à Avaliação de Ambientes Virtuais de Aprendizagem. A Descrição do experimento é apresentada na seção quatro. Os resultados e discussões são descritos na seção cinco. A seção seis é composta das Conclusões e finalmente são apresentadas as Referências.

\section{Ambientes Virtuais de Aprendizagem}

No atual cenário tecnológico é fundamental que a Educação integre-se à sociedade do conhecimento. Uma das formas de realizar essa tarefa é desenvolver estratégias de aprendizagem mediadas por tecnologia da informação (TI), como por exemplo a utilização de AVAs.

Esses Ambientes encontram-se em grande expansão nas mais diversas instituições, como: acadêmicas, empresariais e tecnológicas, com objetivo de ser uma ferramenta de E-learning, possibilitando a capacitação de profissionais e estudantes.

$\mathrm{O}$ conceito de Ambiente Virtual de Aprendizagem, de acordo com Almeida (2004), "relaciona-se à sistemas computacionais, destinados ao suporte de atividades mediadas pelas tecnologias de informação e comunicação”. Estes ambientes permitem integrar múltiplas mídias e recursos, apresentam informações de maneira organizada, proporcionam interações entre pessoas e objetos de conhecimento, visando atingir determinados objetivos.

Os AVAs podem ser empregados como suporte para sistemas de educação a distância, bem como servir de apoio às atividades presenciais de sala de aula e/ou diferentes ambientes por meio da internet ou intranet.

No contexto desta pesquisa foram considerados os AVAs Moodle, TelEduc e Tidia - AE, apresentados nas seções a seguir.

\subsection{Ambiente Virtual de Aprendizagem Moodle}

Conforme os autores Ribeiro e Mendonça (2007), "O AVA Modular Object Oriented Distance Learning (Moodle) é uma plataforma, Open Source, ou seja, pode ser instalado, utilizado, modificado e mesmo distribuído. Seu desenvolvimento objetiva o gerenciamento de aprendizado e de trabalho colaborativo em ambiente virtual, permitindo a criação e administração de cursos on-line, grupos de trabalho e comunidades de aprendizagem". 
Este AVA segundo Garcia e Lacleta (2004), "apresenta estrutura modular, ampla comunidade de desenvolvedores, grande quantidade de documentação, disponibilidade, escalabilidade, facilidade de uso, interoperabilidade, estabilidade e segurança".

\subsection{Ambiente Virtual de Aprendizagem TelEduc}

O AVA TelEduc, segundo Ribeiro e Mendonça (2007),

É uma plataforma Open Source, um ambiente de suporte EAD. O seu desenvolvimento é realizado de acordo com as necessidades, tanto tecnológico como metodológicas, por desenvolvedores do Núcleo de Informática Aplicada à Educação (NIED) da Universidade Estadual de Campinas (UNICAMP).

Em termos de apresentação, o ambiente TelEduc é flexível e está dividido em duas partes: as ferramentas e o conteúdo correspondente à ferramenta selecionada.

\subsection{Ambiente Virtual de Aprendizagem Tidia-Ae}

"O AVA Tidia - Ae é um ambiente colaborativo que gerencia cursos e atividades de aprendizado, dando suporte ao ensino presencial e a distância. O sistema reúne ferramentas de software desenvolvidas especialmente para ajudar alunos, professores, instrutores e pesquisadores em suas ações. Usando um navegador web, os professores podem criar um curso que reúne suas necessidades de aprendizado por meio de um conjunto de ferramentas". Projeto Tidia - AE (2004).

As ferramentas contemplam três grandes grupos: administração, coordenação e comunicação - além de conteúdos para aplicações específicas.

O Projeto Tidia - Ae reúne cerca de quarenta grupos de pesquisa no Estado de São Paulo, é financiado pela (FAPESP) e se subdivide em três subprojetos, um dos quais o Tidia - Ae.

O ambiente é organizado em diferentes áreas de trabalho com distintas funcionalidades, permitindo que os usuários (educadores) possam criar cursos, gerenciálos e participar de maneira colaborativa na execução de trabalhos, tarefas, pesquisas, projetos.

\subsection{Ferramentas}

Considerando os AVAs apresentados, segue a Tabela 1 que apresenta as principais ferramentas comuns aos AVAs analisados:

Tabela 1 - Ferramentas comuns aos ambientes virtuais de aprendizagem

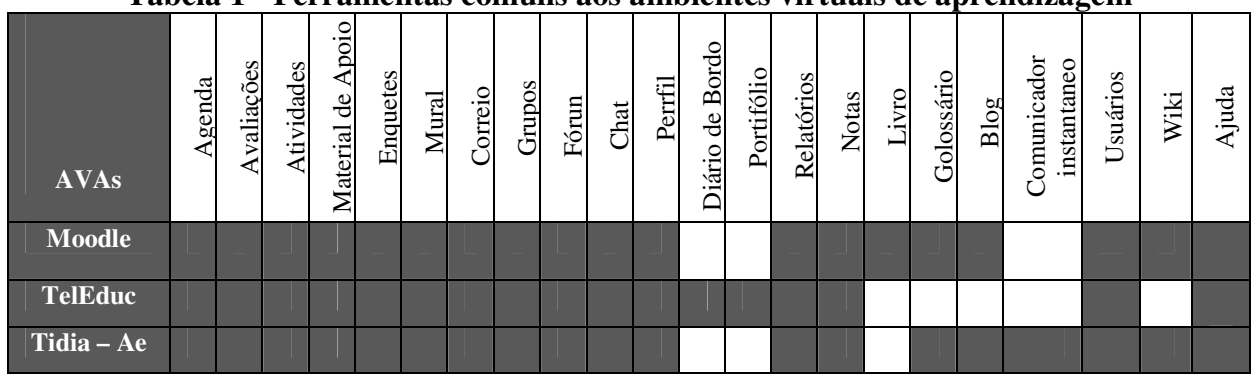

Após analise dos ambientes, observa-se que determinados recursos apresentam nomenclaturas diferentes, porém tem a mesma função. A ferramenta Correio disponibilizada pelo ambiente Moodle se diferencia dos outros, por não permitir o anexo 
de arquivos. No entanto, apresenta a ferramenta Livro, específica para disponibilizar o conteúdo da disciplina no formato de um livro.

Os AVAs Tidia - Ae e TelEduc possuem um recurso de repositório de dados, que permite compartilhar arquivos de forma pública ou privada, diferentemente do AVA Moodle que permite apenas o próprio usuário visualizar seus arquivos.

A ferramenta ajuda está presente nos três ambientes, mas no Moodle, o número de informações disponibilizadas é inferior com relação aos outros ambientes analisados.

\section{Avaliação de Ambientes Virtuais de Aprendizagem}

De acordo com Laguardia "et al."(2007), "avaliar AVAs é uma tarefa complexa, pois além de estarem em constantes estudos e evolução, contemplam variáveis de tecnologia e de aprendizage"..

Os instrumentos de avaliação em AVAs são fundamentais e permitem dar feedback ao desenvolvedor e ao formador sobre os aspectos de usabilidade, ergonomia, confiabilidade, acessibilidade, interação e aspectos pedagógicos.

Para a autora Oliveira (2001),

A acessibilidade, o intuito e a facilidade de uso em um sistema podem ser consideradas como os fatores determinantes para a utilização ou não de um serviço de informação, requerendo constante feedback para que esses serviços possam ser planejados e atendam as necessidades presentes dos seus usuários.

\subsection{Tipos de avaliação de Ambientes Virtuais de Aprendizagem}

Conforme os autores Laguardia “et al."(2007) e Silva (1998),

A avaliação de AVAs pode tomar como base para sua investigação, as condições em que a aprendizagem se realiza (estrutura), os modos pelos quais os estudantes são capazes de interagir sendo apoiados nas suas atividades (processos) e o alcance dos objetivos e das metas propostas (resultados).

De acordo com os autores Filho e Machado (2006),

"Um Ambiente Virtual de Aprendizagem é uma tecnologia educacional que pode ser avaliada sob diferentes aspectos que irão orientar diferentes julgamentos. Para se avaliar diferentes ambientes devem ser levados em conta os paradigmas pedagógicos e ergonômicos que garantem a adequação e a qualidade do processo educacional".

Segundo Valcke e Leeuw (2000),

"A avaliação de AVAs classifica-se em cinco tipos: Avaliação interna e análise do desempenho, Avaliação Ergonômica, Avaliação Externa com enfoque no ambiente sociocultural, Avaliação externa com abordagem dos coordenadores e promotores e o quinto engloba outros métodos de avaliação".

Avaliação interna e análise do desempenho: Neste tipo de avaliação as estratégias mais utilizadas são a construção de indicadores, relacionados à atuação do aluno ao longo do curso e as modificações resultantes da aprendizagem. As estratégias utilizadas são quantitativas (Acessibilidade, Capacidades de buscas, Confiabilidade, Avaliação da plataforma) ou qualitativas (metodologia adotada) com questionários estruturados, semi-estruturados e roteiros para caracterizar satisfação do usuário, participação, interações relacionadas ao uso da tecnologia.

Avaliação Ergonômica Wisner (1995), afirma que:

"A ergonomia é o conjunto dos conhecimentos científicos necessários à concepção de ferramentas, máquinas e dispositivos que possam ser utilizados com conforto, segurança e eficácia. A abordagem ergonômica baseia-se no 
princípio básico de que o trabalho deve se adaptar ao homem. A transferência deste princípio para a informática gerou um enunciado mais específico: adaptar o computador ao usuário, e não o contrário".

Avaliação externa com enfoque no ambiente sócio-cultural: este tipo de avaliação tem como objetivo contemplar análise de custo-benefício e custo-efetividade em que se comparam as experiências de EAD com métodos tradicionais.

Segundo Laguardia "et al."(2007),

"Avaliação externa com abordagem dos coordenadores e promotores: nesta a avaliação, os objetivos de estudo são a variedade de níveis, os conflitos de interesses e o envolvimento dos responsáveis pelos cursos dentro da proposta de EAD digital".

O quinto tipo engloba outros métodos de avaliação, tais como: seleção e uso de mídias adequadas à EAD, relevância e adequação dos cursos e materiais e análise dos escores dos aprendizes.

\subsection{Métodos de avaliação de Ambientes Virtuais de Aprendizagem}

$\mathrm{Na}$ avaliação de AVAs, é necessário dispor de dados sobre características individuais dos participantes (observação do comportamento), do ambiente de aprendizagem, participação, comunicação, materiais e da tecnologia utilizada (Benigno; Trentin, 2000).

A seguir serão apresentados os seguintes métodos de avaliação: questionários (dados quantitativos), observação e entrevista (dados qualitativos). De acordo com Laguardia "et al."(2007), "o uso de questionários é provavelmente o método mais amplamente utilizado nos diversos tipos de avaliação de cursos".

Segundo Dixon (2001),

a aplicação de questionários podem ser realizada de forma presencial ou online, apresentando as seguintes vantagens: rapidez na coleta dos dados, uso de grandes amostras, menor custo de administração e processamento e taxas de retorno mais altas.

A observação dos usuários pode ser de duas formas, direta ou indireta. Na forma direta o avaliador observa o comportamento do usuário, como seqüência de ações. $\mathrm{O}$ usuário pode modificar o comportamento pelo fato de estar sendo observado. $\mathrm{Na}$ observação indireta são coletadas informações de forma automática, não interferindo no comportamento do usuário.

A entrevista é o método que permite coletar opinião do usuário, pois além do desempenho é importante saber o que o usuário pensa sobre o uso que faz da tecnologia.

Para o presente trabalho foram utilizados os tipos e métodos de avaliação descritos na seção quatro.

\section{Descrição do experimento}

A metodologia experimental deste trabalho selecionou cento e trinta usuários, acadêmicos do Curso de Especialização em Tecnologias da Informação e da Comunicação Aplicadas a Educação (TIC) - Sistema Universidade Aberta do Brasil (UAB) e do Programa de Pós-Graduação em Informática (PPGI) - Mestrado em Computação - UFSM.

O experimento consistiu na criação e aplicação do instrumento de avaliação e na observação dos alunos durante a interação com os AVAs Moodle, TelEduc e Tidia - Ae.

Foi analisada a página do Laboratório de Usabilidade (LabIUtil), para escolha das possíveis questões referentes à usabilidade e elaborado o questionário avaliativo a 
partir da análise de três modelos de avaliação: Modelo de Eliane Schlemmer "et al". (2007), Checklist apresentado por Gildásio Guedes (2005) e Andréa Filatro (2004).

No instrumento de avaliação criado, foram utilizados os tipos de avaliação: Avaliação interna e análise do desempenho, Avaliação Ergonômica e outros métodos de avaliação.

Também, foram utilizados os seguintes métodos de avaliação: observação e questionários, apresentados a seguir.

O questionário avaliativo foi construído conforme os seguintes critérios: ferramentas de autoria, ferramentas de interação, ferramentas administrativas, usabilidade, perspectiva didática pedagógica, dados técnicos e dados gerais. Analisou-se sob os seguintes fatores: desempenho da interface de aprendizagem, sincronia e assincronia das comunicações, aspecto indutivo no uso, layout da tela, condução, carga de trabalho, controle explícito, adaptabilidade, gestão de erros, homogeneidade/coerência/consistência, significado dos códigos e denominações e compatibilidade.

$\mathrm{O}$ instrumento desenvolvido consistiu de questões objetivas sobre o ambiente em que as respostas poderiam ser "Sim" (critério existe ou atende as expectativas), "Não" (critério não existe ou não atende as expectativas) e "Não Avaliado" (sem conhecimentos para avaliar o critério). Os avaliadores deveriam marcar apenas uma dessas opções em todas as perguntas. Também foram propostas questões abertas de livre resposta.

A observação foi de forma indireta não interferindo no comportamento dos alunos, sendo assim, coletadas informações de forma automática. Foi realizada através de um acompanhamento dos alunos durante o desenvolvimento de suas atividades e interação com o ambiente por um período de dois meses.

\section{Resultados e Discussão}

A partir dos dados coletados e da análise dos mesmos foram feitas demonstrações gráficas apresentando os resultados obtidos.

Primeiramente é necessário observar que os alunos da pesquisa encontram-se na faixa etária de 31 a 40 anos. Ressalta-se que o AVA Tidia-Ae foi avaliado apenas por alunos do Mestrado, grupo formado por dez integrantes.

Em relação as ferramentas de autoria (Wiki, Enquetes, Agenda, Atividades, Exercícios e Repositório de Dados), constatou-se que o AVA Tidia - Ae apresentou maior número de recursos entre os avaliados. Em contrapartida, por não conter a ferramenta Wiki o AVA TelEduc apresentou maior índice de ferramentas não existentes. (Figura 1). Outro aspecto constatado refere-se à ferramenta agenda, a qual apresenta limitações, comparadas aos demais AVAs.

Constatou-se que entre as ferramentas de interação síncronas e assíncronas, as que mais contribuíram foram correio com $68 \%$ de preferência, seguida de mural e fórum de discussão.

A Figura 2 apresenta a avaliação da usabilidade (navegação rápida, facilidade de utilização, recebimento de feedback, facilidade de envio de tarefas, interface agradável, links em funcionamento e padrão de apresentação coerente). Constatou-se que os AVAs Moodle e TelEduc atingiram um alto grau de satisfação do usuário diante das ferramentas analisadas. 


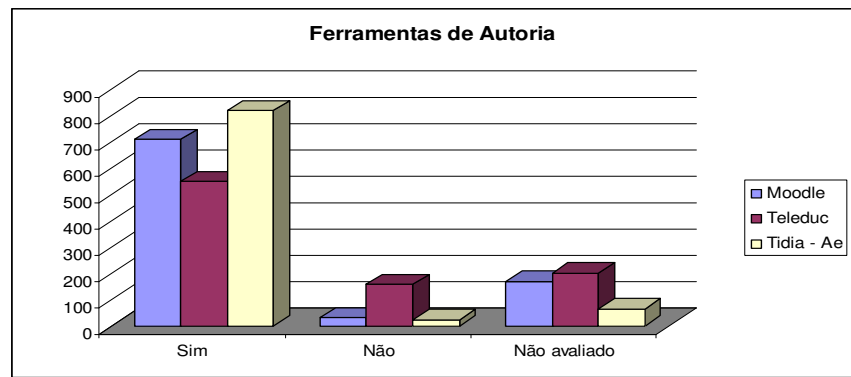

Figura 1 - Ferramentas de autoria

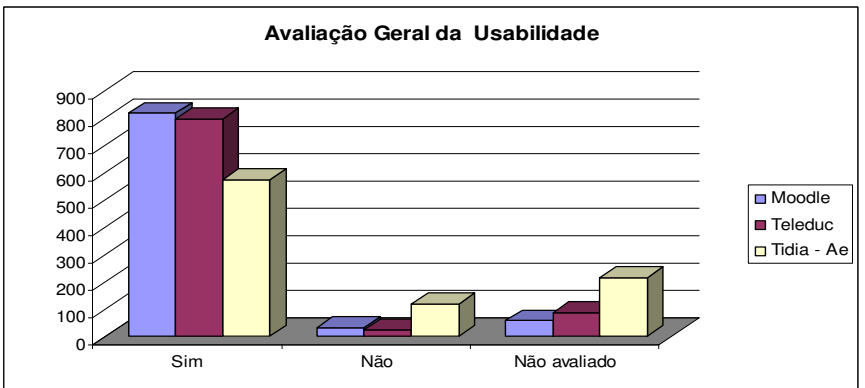

Figura 2 - Avaliação geral da usabilidade

Analisando as ferramentas administrativas (estatísticas de utilização, ferramentas para reaproveitamento de conteúdo, ferramentas de gerenciamento de usuário e ferramentas de registro de avaliações), observou-se que devido à inexperiência dos alunos em relação ao uso de AVAs com o perfil de ADMINISTRADOR, a maioria não avaliou este recurso. A exceção foi dos alunos de Mestrado que, classificaram as ferramentas de reaproveitamento de conteúdo e registro de avaliações como muito boas nos AVAs TelEduc e Tidia - Ae. A ferramenta estatísticas de utilização também, foi considerada muito boa no AVA Moodle.

A partir da análise das características didáticas pedagógicas (ambiente permite o aluno acompanhar as atividades desenvolvidas, permite ao professor e aluno comentar as atividades, atividades propostas em número adequado), apresentadas na Figura 3, constatou-se que o AVA TelEduc ressaltou-se dentre os demais. Um dos motivos refere-se a permissão para alunos comentarem critérios avaliados pelos professores.

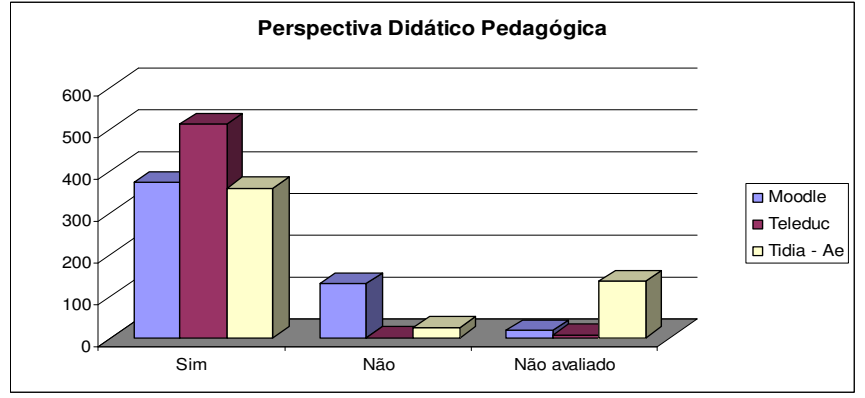

Figura 3 - Perspectiva didática pedagógica

Analisando os aspectos técnicos (segurança no sistema, capacidade de expansão, disponibilidade de recursos para pessoas com necessidades especiais), apresentados na Figura 4, constatou-se que o AVA TelEduc atende aos requisitos expostos acima, seguido do AVA Moodle. Em contrapartida, o AVA Tidia - Ae apresenta um elevado 
índice de itens não avaliado, por ser um AVA desenvolvido recentemente e algumas ferramentas estarem em processo de validação.

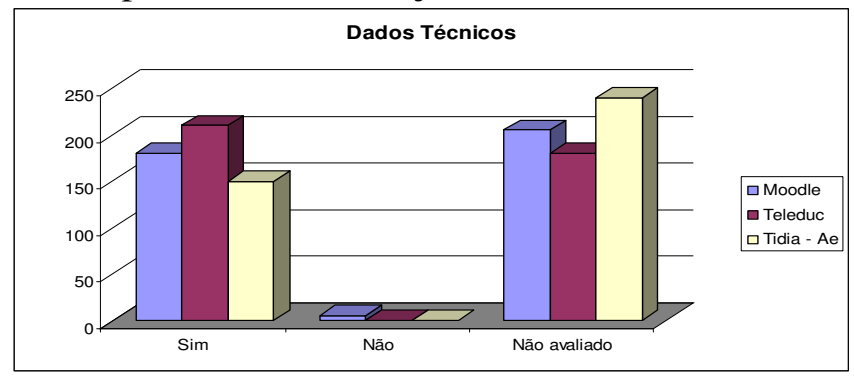

Figura 4 - Dados técnicos

A Figura 5 avaliou Dados Gerais (envio de arquivos nos diversos formatos, suporte ao padrão SCORM, disponibilidade de conteúdo de forma hierárquica), destacando-se, o AVA Moodle, seguido dos AVAs TelEduc e Tidia - Ae.

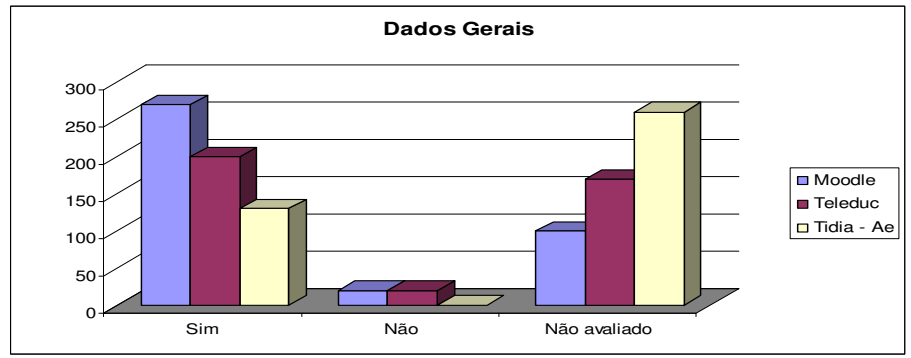

Figura 5 - Dados gerais

Para melhor embasamento, as pesquisa foram feitas por meio de questionamentos aos acadêmicos. Segundo suas opiniões: "Sentimo-nos atraídos em poder nos organizarmos através do ambiente Moodle, aulas mais interativas onde o aluno possa construir sua aprendizagem com acréscimos intelectuais à sua formação e em grupo". (Aluno Mestrado UFSM); "Pareceu-nos que o ambiente TelEduc foi muito bem organizado, disponibilizando um considerável número de recursos, possibilitando a criação de um AVA de qualidade, fácil acesso e navegação com atividades variadas e atraentes" (Aluno TIC - UFSM).

"O AVA Tidia - Ae, contempla grande quantidade de ferramentas, é bem estruturado, adequado ao ensino a distância. (Aluno Mestrado- UFSM).

Outro aspecto abordado foi sobre a avaliação do curso a distancia através dos ambientes Moodle, TelEduc e Tidia - Ae em relação à aprendizagem. As exposições dos alunos foram as seguintes: Os ambientes Moodle, TelEduc e Tidia - Ae são ferramentas que podem ou não contribuir para a aprendizagem, depende do conhecimento que o indivíduo possui sobre seu funcionamento." (Aluno Mestrado UFSM).

Ficou claro nos comentários dos alunos que independente do ambiente utilizado um dos aspectos mais importantes que influenciam no processo de aprendizagem é a interação dos atores envolvidos no processo.

\section{Conclusões}

O instrumento de pesquisa que serviu como ferramenta de avaliação mostrou-se válido e viável, pois através do mesmo, foi realizada a análise e avaliação da estrutura e 
organização dos AVAs Moodle, TelEduc e Tidia - Ae, utilizados pela Universidade Federal de Santa Maria. Foi possível compreender as necessidades dos alunos envolvidos, vislumbrando possíveis melhorias amparadas nas opiniões coletadas.

Quanto à avaliação de adequação e usabilidade, um ponto bastante relevante, em se tratando de ensino a distância, os AVAs analisados proporcionam facilidade de utilização e aprendizagem, são adequados ao ensino-aprendizagem totalmente à distância e servem de apoio ao ensino presencial, tendo como característica várias ferramentas de apoio, destacando-se os AVAs Moodle e TelEduc. Quanto as ferramentas de autoria, o AVA Tidia - Ae apresentou maior número de recursos.

As ferramentas de interação síncronas e assíncronas que mais contribuíram foram correio, seguida de mural e fórum de discussão.

Apenas os alunos de mestrado possuíam experiência com o perfil de administrador de AVAs e suas análises concluíram que as ferramentas de reaproveitamento de conteúdo e registro de avaliações classificam-se como muito boas nos AVAs TelEduc e Tidia - Ae. A ferramenta estatísticas de utilização também, foi considerada muito boa no AVA Moodle.

A partir das análises das características didáticas pedagógicas e aspectos técnicos o AVA TelEduc ressaltou-se dentre os demais principalmente no aspecto referente a permissão de alunos comentarem critérios avaliados pelos professores. Enquanto o AVA Moodle destacou-se na análise dos dados gerais como suporte ao padrão scorm e disponibilidade de conteúdos de forma hierárquica.

Este trabalho almeja contribuir com novos olhares sobre AVAs, seja como elemento auxiliar no complexo processo de escolha de um ambiente, bem como, no suporte para as necessidades de customização dos ambientes em utilização na Instituição, sempre objetivando melhorias para o processo de ensino a distancia, resultando em ambientes que contribuam para a formação integral do acadêmico.

Este trabalho não tem a pretensão de apresentar resultados conclusivos sobre os três ambientes avaliados, mas servir para orientar os trabalhos iniciais no contexto de avaliação de AVAs. Como continuidade do trabalho, a partir dos resultados alcançados, será realizada uma nova rodada de avaliações considerando alterações que se mostraram necessárias, percebidas durante o trabalho, como por exemplo, expansão do instrumento de avaliação e disponibilização de mais tempo para interação e utilização dos AVAs em processo de avaliação principalmente com o perfil de administrador.

\section{Referências}

ALMEIDA, Maria Elizabeth Bianconcini de. (2004). Tecnologia e educação a distância: abordagens e contribuições dos ambientes digitais e interativos de aprendizagem. Disponível em:< http://www.anped.org.br/reunioes/26/trabalhos /ma riaeliza bethalmeida.rtf>. Acesso em: 04 set. 2008.

BENIGNO, V.; TRENTIN, G. The evaluation of online courses. Journal of Computer Assisted Learning, v. 16, p. 56-70, 2000.

DIXON, J. Evaluation tools for flexible delivery (workshop version). Melbourne: TAFE frontiers, 2001.

FILATRO, Andréa. Design instrucional contextualizado: educação e tecnologia. São Paulo: Editora SENAC, 2004

FILHO, Samuel Brasileiro; MACHADO, Elian. Aspectos Metodológicos da Avaliação 
GARCIA, Pablo López; LACLETA, María Luisa Sein-Echaluce. (2004). A revolução pedagógica: o meio Moodle. Disponível em: <http://contenidos.universia .es/html_trad/traducirEspecial/params/especial/bc/seccion/6/titulo/REVOLUCION-

PEDAGOGICA-ENTORNO-MOODLE.html>. Acesso em: 20 ago. 2008. GUEDES, Gildásio. Um checklist para avaliar uma plataforma virtual de aprendizagem. In: ALBUQUERQUE, L. B. (Org). Currículos Contemporâneos: formação, diversidade e identidade em transição. Fortaleza: Editora UFC, 2005.

LAGUARDIA, Josué; PORTELA Margareth Crisóstomo; VASCONCELLOS Miguel Murat . Avaliação em ambientes virtuais de aprendizagem. Educação e Pesquisa. São Paulo, 2007

Laboratório de Usabilidade: Critérios Ergonômicos. Disponível em: < http://www.labiutil.inf.ufsc.br/>. Acesso em 03 jul. 2008.

MOODLE. Modular Object-Oriented Dynamic Learning Environment. Disponível em:< http://moodle.org >. Acesso em 10 ago. 2008.

OLIVEIRA, Elaine Rosangela de. (2001). Avaliação Ergonômica de Interfaces da Scielo - Scientific Electronic Library Online. Disponível em: < http://teses.eps.ufsc.br/defesa/pdf/4705.pdf>. Acesso em: 10 ago. 2008.

PRIMO, Lane. (2008). Auto-Avaliação na Educação a Distância uma alternativa viável. Disponível em: < http://www.prodepa.gov.br/sbc2008/anais/pdf/arq0132.pdf>. Acesso em: 15 jul. 2008.

Projeto TIDIA-Ae: Aprendizado Eletrônico. Disponível em: <http://tidiaae.incubadora.fapesp.br/portal/publicacoes/publications/research\%20assistants\%20repo rts/RelatorioFapespFelipe_junho2005.pdf>. Acesso em: 10 jul. 2008. RIBEIRO, Elvia Nunes; MENDONÇA, Gilda Aquino de Araújo e MENDONÇA, Alzino Furtado. (2007). A importância dos Ambientes Virtuais de Aprendizagem na busca de novos domínios na EAD. Disponível em: < http://www.abed.org.br/congresso2007/tc/4162007104526AM.pdf $>$. Acesso em: 18 ago. 2008.

TELEDUC - Educação a distância. Disponível em:< http://teleduc.nied.unicamp.br/teleduc/ >. Acesso em: 10 jul. 2008.

SCHLEMMER, Eliane; SACCOL, Amarolinda; GARRIDO, Susane. (2007). Um Modelo Sistêmico de Avaliação de Softwares para Educação a Distância como apoio à Gestão de EAD. Disponível em: < http://www.ead.fea.usp.br/cadpesq/arquivos/493.pdf.>. Acesso em: 08 set. 2008.

SILVA, Cassandra Ribeiro de Oliveira. Bases pedagógicas e ergonômicas para consepção e avaliação de produtos educacionais informatizados. Programa de PósGraduação em Engenharia da Produção. Florianópolis, 1998.

Universidade Aberta do Brasil. Disponível em: < http://uab.capes.gov.br/index.php >. Acesso em:15 abr. 2008.

VALCKE, M. M., LEEUW, F. L. Evaluating digital distance learning programs and activities. Washington: World Bank Institute, 2000.

WISNER, Alain. (1995). As transformações do trabalho e os desafios teóricosmetodológicos da ergonomia: Desafios teóricos metodológicos da ergonomia. Disponível em: <http://www.unb .br/ip/labergo/sitenovo /Julia/Artigos/paraosite /DTM E.PDF>. Acesso em: 25 ago. 2008. 